\title{
Effects of dexamethasone and meloxicam on Borrelia burgdorferi-induced inflammation in glial and neuronal cells of the central nervous system
}

\author{
Geeta Ramesh ${ }^{1 \dagger}$, Alejandra N. Martinez ${ }^{1+}$, Dale S. Martin ${ }^{1}$ and Mario T. Philipp ${ }^{1,2^{*}}$
}

\begin{abstract}
Background: Lyme neuroborreliosis (LNB), caused by the spirochete Borrelia burgdorferi $(\mathrm{Bb})$, affects both the central and peripheral nervous systems. Previously, we reported that in a model of acute LNB in rhesus monkeys, treatment with the anti-inflammatory drug dexamethasone significantly reduced both pleocytosis and levels of cerebrospinal fluid (CSF) immune mediators that were induced by Bb. Dexamethasone also inhibited the formation of inflammatory, neurodegenerative, and demyelinating lesions in the brain and spinal cord of these animals. In contrast, these signs were evident in the infected animals that were left untreated or in those that were treated with meloxicam, a non-steroidal anti-inflammatory drug.

Methods: To address the differential anti-inflammatory effects of dexamethasone and meloxicam in the central nervous system (CNS), we evaluated the potential of these drugs to alter the levels of Bb-induced inflammatory mediators in culture supernatants of rhesus frontal cortex (FC) explants, primary rhesus astrocytes and microglia, and human oligodendrocytes. We also ascertained the potential of dexamethasone to modulate Bb-induced apoptosis in rhesus FC explants. As meloxicam is a known COX-2 inhibitor, we evaluated whether meloxicam altered the levels of COX-2 as induced by live Bb in cell lysates of primary rhesus astrocytes and microglia.

Results: Dexamethasone but not meloxicam significantly reduced the levels of several Bb-induced immune mediators in culture supernatants of FC explants, astrocytes, microglia, and oligodendrocytes. Dexamethasone also had a protective effect on Bb-induced neuronal and oligodendrocyte apoptosis in rhesus FC explants. Further, meloxicam significantly reduced the levels of Bb-induced COX-2 in microglia, while both $\mathrm{Bb}$ and meloxicam were unable to alter the constitutive levels of COX-2 in astrocytes.

Conclusions: These data indicate that dexamethasone and meloxicam have differential anti-inflammatory effects on Bb-induced inflammation in glial and neuronal cells of the CNS and help explain the in vivo findings of significantly reduced inflammatory mediators in the CSF and lack of inflammatory neurodegenerative lesions in the brain and spinal cord of Bb-infected animals that were treated with dexamethasone but not meloxicam. Signaling cascades altered by dexamethasone could serve as possible therapeutic targets for limiting CNS inflammation and tissue damage in LNB.
\end{abstract}

Keywords: Lyme neuroborreliosis, Borrelia burgdorferi, Inflammation, Apoptosis, Dexamethasone, Meloxicam

\footnotetext{
* Correspondence: philipp@tulane.edu

†Equal contributors

'Division of Bacteriology and Parasitology, Tulane National Primate Research

Center, Covington, LA, USA

${ }^{2}$ Department of Microbiology and Immunology, Tulane University Medical

School, New Orleans, LA, USA
} 


\section{Background}

Lyme disease is caused by infection with the spirochete Borrelia burgdorferi (Bb) [1]. The nervous system involvement in Lyme disease, called Lyme neuroborreliosis (LNB), may affect both the central and peripheral nervous systems in about 15\% of Lyme disease patients. Symptoms of acute LNB include painful meningoradiculitis with inflammation of dorsal nerve roots and lancinating, radicular pain (Bannwarth's syndrome), lymphocytic meningitis, and various forms of cranial or peripheral neuritis [2].

The rhesus macaque is the most accurate model of human LNB [3-6]. Previously, we reported that leptomeningitis and radiculitis that manifest in monkeys with acute LNB are concomitant with the inflammatory meditator response elicited by $\mathrm{Bb}$ [6]. Importantly, lymphocyte and plasma cell infiltration in the leptomeninges and perivascular infiltrates of immune cells adjacent to white matter lesions in the brain and transverse myelitis lesions in the spinal cord have been documented in pathological examinations of lesions from cases of human LNB $[2,7,8]$.

We hypothesized that $\mathrm{Bb}$ induces the production of inflammatory mediators in glial and neuronal cells and that this response has a role in potentiating glial and neuronal apoptosis. We recently explored if inflammation had a causal role in mediating the pathogenesis of LNB by evaluating the inflammatory changes in rhesus macaques infected with $\mathrm{Bb}$ that were left untreated or were given either the anti-inflammatory drug dexamethasone, a steroid that inhibits the expression of several immune mediators [9], or meloxicam, a non-steroidal anti-inflammatory drug that inhibits cyclooxygenase-2 (COX-2) [10]. Dexamethasone treatment significantly reduced the levels of several cytokines and chemokines, and pleocytosis in the CSF, and prevented inflammatory and/or neurodegenerative and demyelinating lesions in the central and peripheral nervous systems [11]. Conversely, infected animals that were treated with meloxicam showed similar levels of immune mediators in the CSF and displayed similar lesions in the CNS and PNS to those seen in infected animals that were left untreated. Also, the effects of these drugs in neuronal cultures of dorsal root ganglia and of myelinating cells of the PNS infected with Bb showed that dexamethasone but not meloxicam significantly reduces the levels of apoptosis and those of several cytokines and chemokines [12].

In this study, we evaluate the effects of these drugs on $\mathrm{Bb}$-induced inflammation in glial and neuronal cells of the CNS. Results show that dexamethasone but not meloxicam significantly reduces the levels of several cytokines and chemokines as induced by live $\mathrm{Bb}$ in rhesus astrocytes, microglia, and FC explants, in addition to human oligodendrocytes. Likewise, dexamethasone showed a protective effect on cell death, as both neurons and oligodendrocytes evinced reduced apoptosis in rhesus FC explants. Further, meloxicam was able to significantly reduce the levels of $\mathrm{Bb}$-induced $\mathrm{COX}-2$ in rhesus microglia, while it did not alter the constitutive levels of COX2 in rhesus astrocytes. These data indicate that as with PNS cells [13], dexamethasone and meloxicam have differential anti-inflammatory effects on Bb-induced inflammation in glial and neuronal cells of the CNS.

\section{Methods}

\section{Growth and preparation of live spirochetes}

B. burgdorferi B31 clone 5A19 spirochetes, passage 1-3, were grown in Barbour-Stoenner-Kelly (BSK) medium, supplemented with $6 \%$ rabbit serum (Sigma, St. Louis, $\mathrm{MO}$ ) and antibiotics (rifampicin at $45.4 \mu \mathrm{g} / \mathrm{mL}$, phosphomycin at $193 \mu \mathrm{g} / \mathrm{mL}$, and amphotericin at $0.25 \mu \mathrm{g} /$ $\mathrm{mL}$ ) to late logarithmic phase under microaerophilic conditions. Spirochetes were pelleted, washed using sterile phosphate-buffered saline (PBS), and resuspended in the working medium at the desired density, as previously described [12].

Frontal cortex brain (FC) explant experiments: incubation of brain slices with B. burgdorferi (Bb), dexamethasone, and meloxicam for collection of culture supernatants for multiplex ELISA assays and apoptosis assays with tissue explants

Freshly harvested frontal cortex tissues were collected at necropsy from three rhesus macaques (Macaca mulatta) that were scheduled for euthanasia due to chronic idiopathic diarrhea or had undergone trauma. Animals were euthanized in accordance with the recommendations of the American Veterinary Medical Association's Panel on Euthanasia. The frontal cortex was sliced into 2-mm sections, and each section was placed in separate wells of 12-well plates. Each well contained $2 \mathrm{~mL}$ of RPMI 1640 medium (BioWhittaker, Walkersville, MD) supplemented with $10 \%$ FBS, as previously described [14]. Tissue sections were exposed to medium alone or to medium with added $\mathrm{Bb}\left(1 \times 10^{7}\right.$ bacteria/mL $)$ in the presence or absence of either $150 \mu \mathrm{M}$ dexamethasone (Sigma) or $100 \mu \mathrm{M}$ meloxicam (Sigma). Controls with no spirochetes were also included. Prior to stimulation with live $\mathrm{Bb}, \mathrm{FC}$ explant cultures were incubated with the respective concentrations of dexamethasone or meloxicam for $1 \mathrm{~h}$ in a humidified $5 \% \mathrm{CO}_{2}$ incubator at $37{ }^{\circ} \mathrm{C}$, after which they were washed and then incubated in fresh growth medium containing the appropriate concentrations of dexamethasone or meloxicam and live $\mathrm{Bb}$ for an additional $6 \mathrm{~h}$ at $37^{\circ} \mathrm{C}$. The above concentrations of dexamethasone and meloxicam were the highest concentrations that were found to be safe as evaluated by the trypan blue exclusion assay (Sigma) in previous 
studies with cultures of human Schwann cells and rhesus dorsal root ganglia cultures [13]. At the end of the incubation times, culture supernatants were collected for evaluation of inflammatory mediators. Culture supernatants were centrifuged at $4{ }^{\circ} \mathrm{C}$ at $2000 \times g$ to remove any suspended bacteria, and the supernatant was aliquoted and stored at $-70{ }^{\circ} \mathrm{C}$ until used, as previously reported [13]. Tissue explants in respective wells were fixed using $2 \%$ paraformaldehyde and cryopreserved for apoptosis assays.

\section{Primary cultures of rhesus astrocytes and microglia}

Primary rhesus astrocyte and microglial cultures were established following previously described protocols [15]. Briefly, after the meninges and blood vessels from fresh frontal cortex tissue were removed, tissue was mechanically dissociated, treated with $0.25 \%$ trypsin, $0.38 \mathrm{~g} / \mathrm{mL}$ EDTA (Invitrogen), and 0.1\% DNase (SigmaAldrich), and incubated at $37{ }^{\circ} \mathrm{C}$ for 40 min with intermittent shaking. After incubation, the dissociated tissue was centrifuged for $10 \mathrm{~min}$ at $425 \times g$ and cells filtered through a Nitex filter $(20 \mu \mathrm{m})$ and resuspended in glial culture medium, which was composed of DMEM-F-12 with L-glutamine and HEPES buffer, $10 \%$ fetal bovine serum (HyClone), $0.5 \mathrm{ng} / \mathrm{mL}$ of granulocyte-macrophage colony-stimulating factor (Sigma-Aldrich), $100 \mathrm{U} / \mathrm{mL}$ penicillin, and $100 \mu \mathrm{g} / \mathrm{mL}$ streptomycin. After 14 to 21 days in culture at $37{ }^{\circ} \mathrm{C}$, dislodged microglia were collected after vigorously tapping the flasks, and resuspended in glial culture medium.

To obtain purified astrocytes, glial cells were incubated for $90 \mathrm{~min}$ in $10 \mathrm{mM} \mathrm{L}$-leucine methyl ester (LME) (Sigma-Aldrich). This concentration has been shown to be effective in ensuring maximal microglial lysis with minimal toxicity to astrocytes [15]. Thereafter, astrocytes were washed thoroughly and resuspended in glial culture medium. Purity of astrocytes and microglial cultures was assessed by staining with a specific microglial marker anti-IBA antibody at a concentration of 1:50 (Wako Chemicals, Richmond, VA) and was routinely of $99 \%$. Astrocyte cultures were stained with astroglial marker anti-GFAP antibody at a dilution of 1:200 (Sigma) after permeabilizing fixed astrocyte cultures with a mixture of chilled ethanol-acetic acid mixture $(2: 1)$, for $5 \mathrm{~min}$ at $-20{ }^{\circ} \mathrm{C}$, for detection of intracellular phenotypic markers as previously described [16]. Confocal microscopy was performed using a Leica TCS SP8 confocal microscope (Leica Microsystems, Exton, PA).

\section{Human oligodendrocyte cultures}

The human oligodendrocyte cell line MO3.13 obtained from CELLutions Biosystems Inc. (Burlington, Ontario, Canada) was revived and established following manufacturer's instructions as previously described [17].
Differentiated oligodendrocytes were obtained after maintaining cultures in differentiation medium (DM), consisting of DMEM, $\mathrm{P} / \mathrm{S}$, and phorbol 12-myristate 13-acetate (Sigma), at a concentration of $100 \mathrm{nM}$ and devoid of serum.

\section{Evaluating the anti-inflammatory potential of dexamethasone and meloxicam in rhesus astrocytes and microglia and human oligodendrocyte cultures stimulated with live $\mathrm{Bb}$}

Glial and $\mathrm{Bb}$ cultures were washed in their respective media, devoid of antibiotics. Controls with no spirochetes were also included. Prior to stimulation with live $\mathrm{Bb}$, glial cell cultures were incubated with various concentrations of dexamethasone $5 \mu \mathrm{M}, 15 \mu \mathrm{M}$ and $150 \mu \mathrm{M}$ (Sigma) or meloxicam $1 \mu \mathrm{M}, 10 \mu \mathrm{M}, 50 \mu \mathrm{M}$ and $100 \mu \mathrm{M}$ (Sigma) for $2 \mathrm{~h}$ at $37^{\circ} \mathrm{C}$, after which they were washed and then incubated in fresh growth medium containing the respective concentrations of dexamethasone or meloxicam and live $\mathrm{Bb}$ at a multiplicity of infection (MOI) of $10: 1$ at $37^{\circ} \mathrm{C}$. The effect of the carrier substance of dexamethasone (2-hydroxypropyl)- $\beta$-cyclodextrin), at the respective molar concentrations accompanying dexamethasone, was assessed by incubating rhesus astrocytes and microglial cultures in the presence and absence of $\mathrm{Bb}$ and carrier alone at 15, 45, and $450 \mu \mathrm{M}$, respectively, as previously described for oligodendrocyte cultures [16]. After $48 \mathrm{~h}$, culture supernatants were collected and processed for evaluation of inflammatory mediators. The drug concentrations were chosen based on previous reports where dexamethasone $(5-150 \mu \mathrm{M})$ had been shown to inhibit the production of CCL2 in mice microglia [18], and meloxicam $(1-100 \mu \mathrm{M})$ had been shown to be effective in inhibiting COX-2 in vitro [19].

Evaluation of immune mediators from culture supernatants The concentrations of cytokines and chemokines present in the culture supernatants from rhesus astrocyte and microglial cultures and rhesus FC explant cultures were quantified using the MilliPlex MAP Non-Human Primate Cytokine Magnetic Bead Panel-Premixed 23 Plex, Cytokine-Chemokine Array kit (Millipore), following the manufacturer's instructions. The concentrations of cytokines and chemokines present in the culture supernatants from human oligodendrocytes were quantified using the Human 14-plex Cytokine-Chemokine Array kit (Millipore), following the manufacturer's instructions. The multiplex plate was read using a Bio-Plex 200 Suspension Array Luminex System (Bio-Rad, Hercules, CA, USA).

Apoptosis by in situ TUNEL assay and confocal microscopy Tissue sections from rhesus FC explants were incubated with anti-NeuN or anti-S-100 antibodies and then subjected to the TUNEL-ApopTagPlus fluorescein in situ apoptosis assay (Chemicon, Temecula, CA) as previously 
described [14]. The percentage of apoptotic neurons and the percentage of apoptotic oligodendrocytes in brain sections were evaluated by counting at least 500 cells, followed by the percentage of cells that showed colocalization of both the terminal deoxynucleotidyl transferase dUTP nick end labeling (TUNEL) signal and NeuN or S-100 expression, respectively. Apoptotic cells from ten fields were counted from each section for the various incubation conditions. All counts were made by viewing slides under a fixed magnification of $\times 63$ (corresponding to an area of $0.05 \mathrm{~mm}^{2}$ ) using the Leica TCS SP8 confocal microscope. The identity of the oligodendrocytes that stained with the anti-S-100 antibody was confirmed by their morphology as previously described [14].

\section{COX-2 activity assay from culture lysates}

Astrocytes and microglia cell lysates were prepared following the manufacturer's instructions described in the COX Activity Assay Kit (Cayman Chemicals, Ann Arbor, MI). The Cayman's COX activity assay kit measures the total peroxidase activity of COX in cell lysates and is assayed colorimetrically by monitoring the appearance of oxidized $\mathrm{N}, \mathrm{N}, \mathrm{N}^{\prime}, \mathrm{N}^{\prime}$-tetramethyl-p-phenylenediamine (TMPD) at $590 \mathrm{~nm}$. The total COX-2 activity in the sample was calculated using the formula provided by the manufacturer.

\section{Statistical evaluation}

The two-way ANOVA and Tukey's multiple comparison test was used to evaluate the statistical significance between means of data sets, using Graphpad Prism software (Graph Pad Software Inc.) version 5.

\section{Results}

Effects of dexamethasone and meloxicam on the levels of cytokines and chemokines induced by $\mathrm{Bb}$ in rhesus frontal cortex explants

Culture supernatants from slices of the frontal cortex that were incubated with $\mathrm{Bb}$ and treated with dexamethasone resulted in significantly reduced levels of IL6, IL-8, CCL2, IL-1 $\beta$, IL-18, TNF- $\alpha$, VEGF, and G-CSF, compared with slices that were incubated with $\mathrm{Bb}$ alone. In contrast, results from meloxicam treatment showed that this non-steroidal drug was unable to significantly reduce the levels of the pro-inflammatory cytokines and chemokines evaluated (Fig. 1a-i). The levels of IL-10, a cytokine known to counteract pro-inflammatory mediators, were also measured, and results showed that there were no significant differences after treatment with either one of the anti-inflammatory drugs (Fig. 1e). However, the level of IL-10 was lower after dexamethasone treatment than it was with $\mathrm{Bb}$ alone, albeit not significantly. The pattern of mediator response found in the tissue section supernatants was also present in the tissue lysates (data not shown).

Effects of dexamethasone and meloxicam on the levels of CCL2 induced by $\mathrm{Bb}$ in cultures of rhesus astrocytes and microglia and human oligodendrocytes

A mediator predominantly released by microglia in vitro and into the CSF of rhesus macaques with Lyme meningitis is the chemokine CCL2 $[16,20]$. Therefore, we evaluated the ability of different concentrations of dexamethasone and meloxicam to reduce CCL2 levels induced by $\mathrm{Bb}$ in cultures of primary rhesus astrocytes and microglia, as well as human oligodendrocytes after $48 \mathrm{~h}$ of incubation. As expected, dexamethasone (Fig. 2b, e, h) but not meloxicam (Fig. 2c, f, i) significantly reduced CCL2 levels at the drug concentrations tested. Moreover, the antiinflammatory effect of the dexamethasone formulation was confirmed to be due to its dexamethasone fraction and not due to the carrier substance (2-hydroxypropyl)- $\beta$ cyclodextrin (HPC) (Fig. 2b, e, h).

The effect of these two anti-inflammatory drugs was also assessed using a multiplex analysis of cytokines and chemokines that were released from the studied glial cells in the presence of Bb. Table 1 summarizes these results and shows that multiple mediators are significantly affected by dexamethasone but not by meloxicam.

\section{Dexamethasone protects FC neurons and oligodendrocytes from $\mathrm{Bb}$-induced apoptosis}

Live $\mathrm{Bb}$ induced enhanced levels of apoptosis, as measured by the TUNEL assay, in both neurons and oligodendrocytes as compared to medium alone. A representative confocal image of apoptosis in the presence of live $\mathrm{Bb}$, as visualized by the in situ TUNEL assay, and a graph of the quantification levels of dexamethasone protection in neurons are shown in Fig. 3a, b and in oligodendrocytes in Fig. 3c, d.

\section{Meloxicam affects levels of $\mathrm{COX}-2$ induced by $\mathrm{Bb}$ in in vitro cultures of rhesus microglia but not of astrocytes} COX-2 is known to be involved in both inflammation and apoptosis in brain disorders [21]. As meloxicam is a known COX-2 inhibitor, we evaluated whether it was capable of altering the levels of COX-2 induced by live $\mathrm{Bb}$ in cell lysates of rhesus astrocytes and microglia. Results showed that at a concentration within the range used in our assays, this non-steroidal anti-inflammatory drug had an effect in microglia but not in astrocytes (Fig. 4). However, COX-2 appears to be constitutively expressed in astrocytes (Fig. 4b). Thus, meloxicam significantly reduced $\mathrm{COX}-2$ levels as induced by $\mathrm{Bb}$ in rhesus microglia (Fig. 4a) but was unable to reduce the levels of pro-inflammatory cytokines and chemokines (Fig. 2c, f, i). These results suggest a cell type differential 

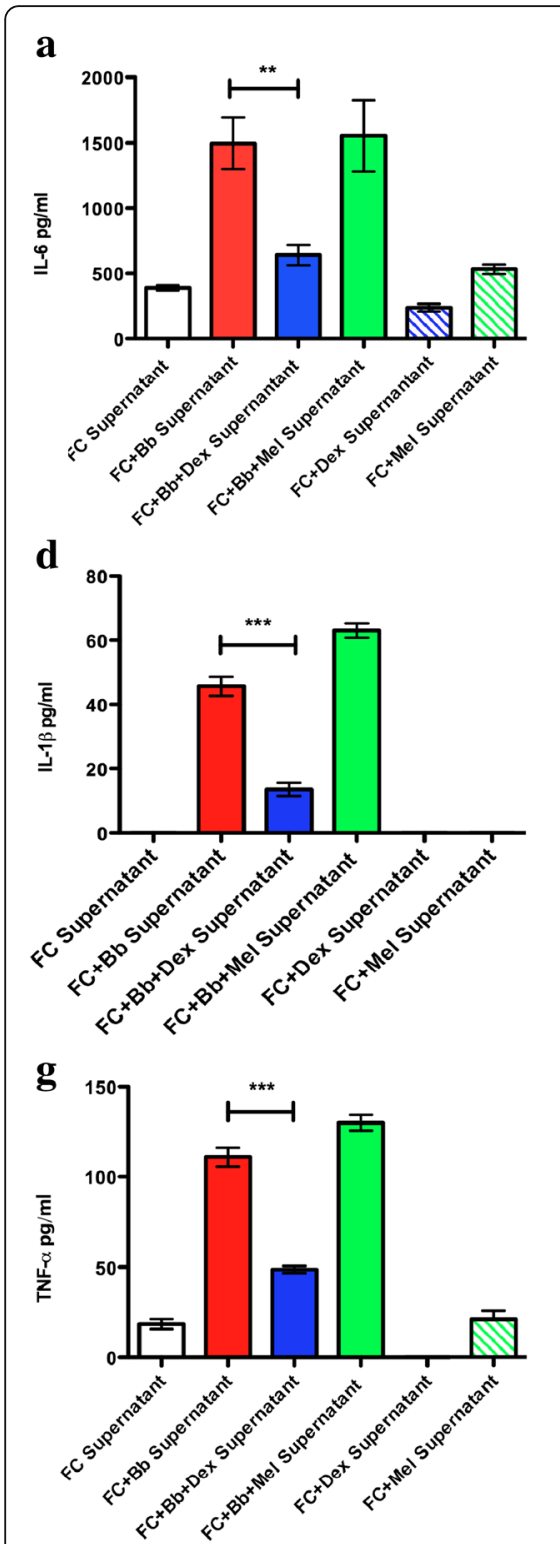
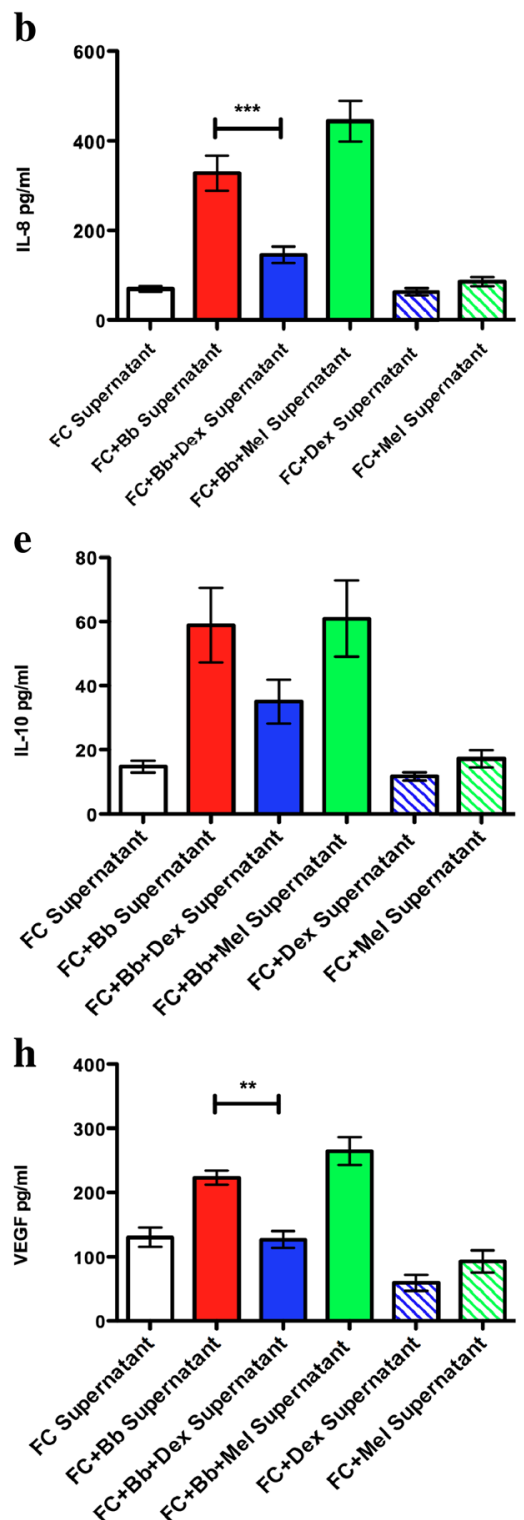

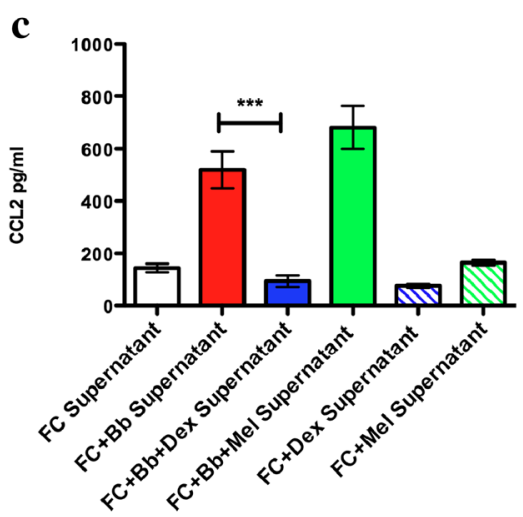

f

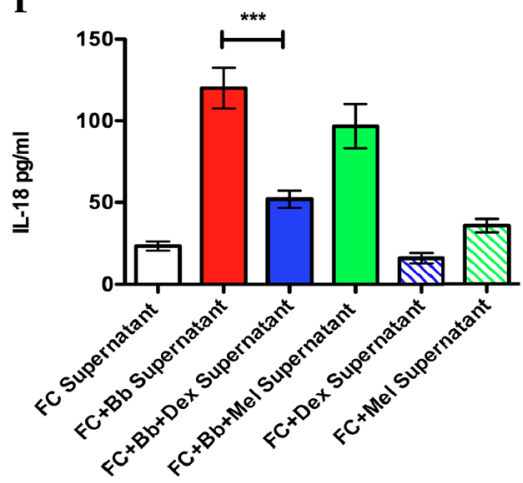

i

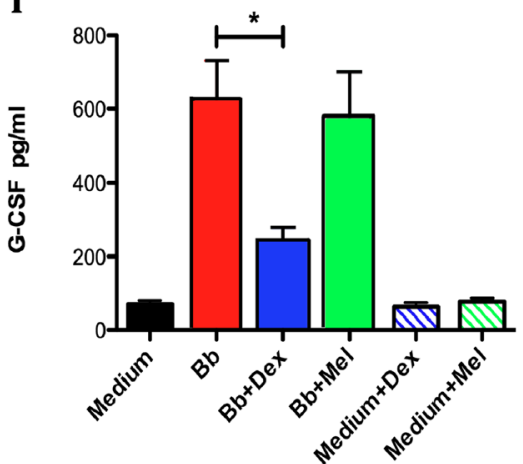

Fig. 1 Dexamethasone but not meloxicam reduces the levels of Bb-induced cytokines and chemokines in frontal cortex explants after $6 \mathrm{~h}$ of incubation. The graphs represent the effect of the anti-inflammatory drugs on the levels of a $\|L-6, \mathbf{b}\| L-8, \mathbf{c} C C L 2, \mathbf{d}\|L-1 \beta, \mathbf{e}\| L-10, \mathbf{f} \| L-18, \mathbf{g} T N F-a$, $\mathbf{h}$ VEGF, and $\mathbf{i}$ G-CSF. The two-way ANOVA and Tukey's multiple comparison test were used to evaluate the statistical significance between means and SEM of triplicate data sets, ${ }^{*} p<0.05,{ }^{* *} p<0.01,{ }^{* * *} p<0.001$

expression of COX-2 and demonstrate that meloxicam is able to exert its effects in specific cell targets where COX-2 levels are upregulated by $\mathrm{Bb}$.

\section{Discussion}

Once inside the CNS, Bb spirochetes elicit inflammatory mediators from glial and neuronal cells, as well as lymphomonocytic pleocytosis in the CSF [11, 12, 16, 22, 23]. Research models have allowed insights into the pathogenesis of LNB, and although several studies suggest that inflammation is required to trigger apoptosis of neurons and glial cells, a causal relationship between these two phenomena had not been demonstrated. Recently, we addressed the hypothesis that inflammation is a key factor in LNB pathogenesis by using two well-known antiinflammatory drugs, dexamethasone and meloxicam, in a rhesus macaque model. Results revealed that dexamethasone but not meloxicam treatment effectively suppressed inflammation due to exposure to $\mathrm{Bb}$, and this resulted in inhibition of glial and neuronal damage [11]. To corroborate this finding, our in vitro studies using PNS cells, namely, primary DRG neurons and human Schwann cell cultures also explored the effects of such drugs in the presence of $\mathrm{Bb}$ [13]. Dexamethasone and 

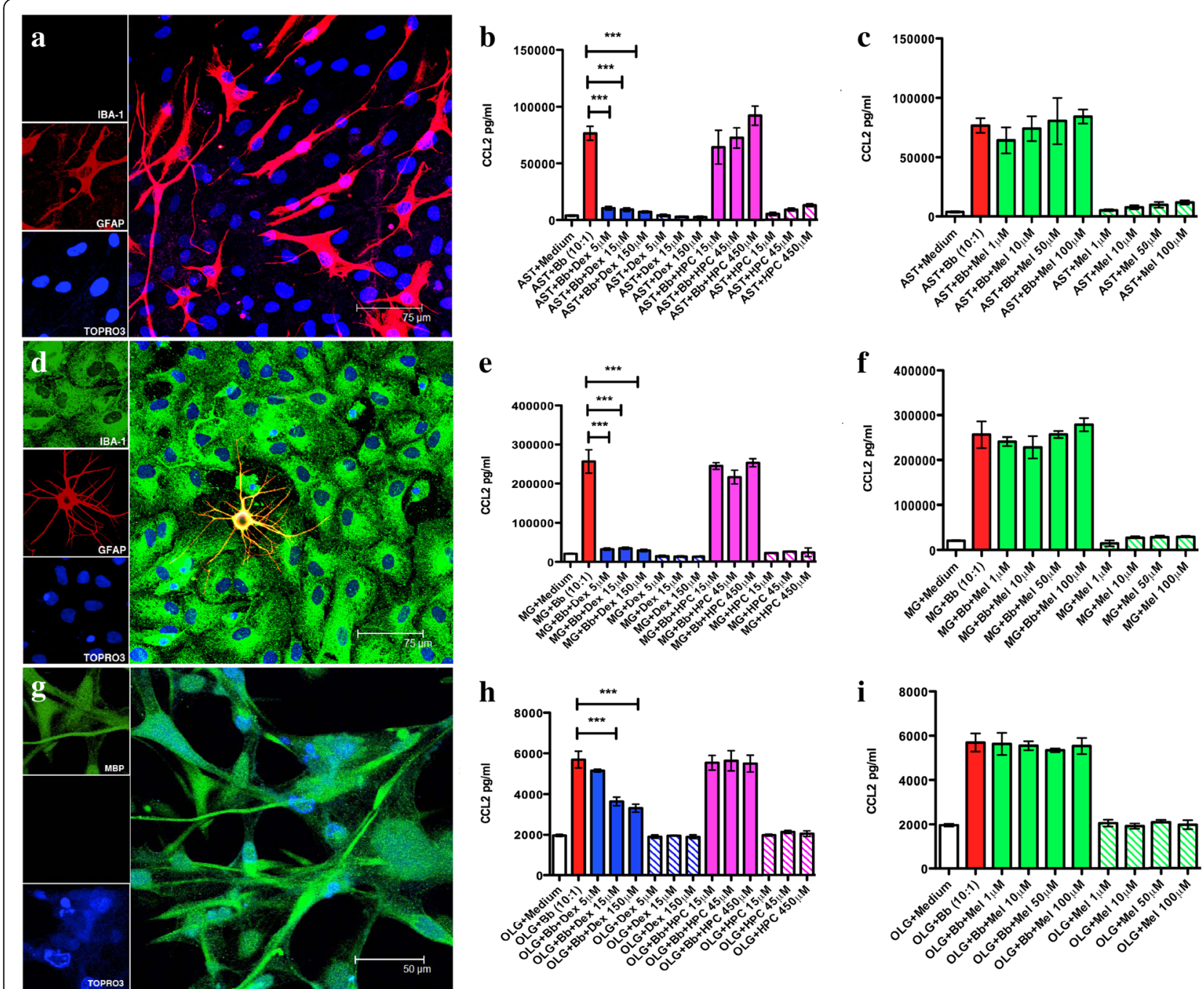

Fig. 2 Dexamethasone but not meloxicam affects the levels of CCL2 induced by live Bb at a multiplicity of infection of 10:1 in in vitro cultures of rhesus astrocytes $(\mathbf{a}-\mathbf{c})$, rhesus microglia $(\mathbf{d}-\mathbf{f})$, and human oligodendrocytes $(\mathbf{g}-\mathbf{i})$ after $48 \mathrm{~h}$ of incubation. The effect of different concentrations of dexamethasone $(\mathbf{b}, \mathbf{e}, \mathbf{h})$ and meloxicam $(\mathbf{c}, \mathbf{f}, \mathbf{i})$ on the levels of CCL2 as induced by live Bb in different glial cells are represented in the graphs. Representative confocal micrographs showing cell morphology and cell culture purity of a primary rhesus astrocytes (GFAP, red), $\mathbf{b}$ primary rhesus microglia (IBA-1, green), and $\mathbf{c}$ human oligodendrocytes (MBP, green). The nuclei of all cells in $\mathbf{a}-\mathbf{c}$ appear blue due to staining with the nuclear stain TOPRO3. The two-way ANOVA and Tukey's multiple comparison test were used to evaluate the statistical significance between means and SEM of triplicate data sets for astrocytes and microglia, and the mean and SD from two assessments of human oligodendrocytes, ${ }^{* * *} p<0.001$

meloxicam demonstrated to have differential antiinflammatory effects, with dexamethasone being the only one able to significantly reduce Bb-induced immune mediators and apoptosis of PNS cells.

Here, we assessed the ability of dexamethasone and meloxicam to affect inflammation and apoptosis in CNS cells and tissues. To this end, we evaluated the levels of $\mathrm{Bb}$-induced inflammatory mediators in culture supernatants of rhesus FC explants (Fig. 1) and purified primary rhesus astrocytes and microglia and human oligodendrocytes (Fig. 2). We also ascertained the potential of dexamethasone to modulate Bb-induced neuronal and oligodendrocyte apoptosis in rhesus FC explants (Fig. 3).
The compiled results from this study help to explain our in vivo findings of significantly reduced inflammatory mediators in the CSF and lack of inflammatory neurodegenerative lesions in the brain and spinal cord of Bb-infected animals that were treated with dexamethasone [11].

The $\mathrm{Bb}$ genome encodes, approximately, 150 lipoproteins that play an important role in disease pathogenesis and host immunity [24]. The majority of the proinflammatory lipoproteins are outer surface proteins, and their differential expression in various tissues and at different times during infection appear to be critical determinants of disease $[17,25]$. An effective host response 
Table 1 Cytokines and chemokines are induced by Bb in glial cells of the CNS and significantly reduced by dexamethasone (when used at concentrations of $150 \mu \mathrm{M} ; p<0.001$ ), as evaluated in culture supernatants after $48 \mathrm{~h}$. Meloxicam had no effect

\begin{tabular}{|c|c|c|c|c|c|c|c|c|}
\hline \multicolumn{3}{|l|}{ Rhesus astrocytes } & \multicolumn{3}{|l|}{ Rhesus microglia } & \multicolumn{3}{|c|}{ Human oligodendrocytes } \\
\hline Mediator pg/mL & $\mathrm{Bb}$ & $\mathrm{Bb}+\mathrm{dex}$ & Mediator pg/mL & $\mathrm{Bb}$ & $\mathrm{Bb}+\mathrm{dex}$ & Mediator pg/mL & $\mathrm{Bb}$ & $\mathrm{Bb}+\mathrm{dex}$ \\
\hline$\overline{I L-1 \beta}$ & 24 & 0 & $\mathrm{IL}-1 \beta$ & $64 \pm 10$ & 0 & IL-6 & $141 \pm 1.2$ & $65 \pm 2.7$ \\
\hline $\mid \mathrm{L}-2$ & 146 & $21.8 \pm 10$ & $\mathrm{IL}-2$ & $268 \pm 12$ & 54 & IL-8 & $1145 \pm 609$ & $97 \pm 2.2$ \\
\hline IL-6 & $27175 \pm 2567$ & $596 \pm 51$ & IL-6 & $92562 \pm 2979$ & $1319 \pm 77$ & CCL2 & $5691 \pm 410$ & $1898 \pm 96$ \\
\hline |L-8 & $113486 \pm 882$ & $1636 \pm 40$ & IL-8 & $493804 \pm 3550$ & $3069 \pm 309$ & & & \\
\hline IL-10 & 36 & 3.72 & IL-10 & $40.45 \pm 6.26$ & 17.94 & & & \\
\hline CCL2 & $76594 \pm 1076$ & $2442 \pm 260$ & CCL2 & $256397 \pm 2581$ & $12415 \pm 409$ & & & \\
\hline CCL3 & 95.43 & 0 & CCL3 & $2948 \pm 176$ & $136 \pm 10$ & & & \\
\hline CCL4 & 25.34 & 0 & CCL4 & 183.64 & 0 & & & \\
\hline VEGF & $1220 \pm 122$ & 0 & VEGF & $3153 \pm 108$ & $941 \pm 28$ & & & \\
\hline \multirow[t]{2}{*}{ GMCSF } & $674 \pm 20$ & 0 & GMCSF & $3076 \pm 127$ & $501 \pm 6.14$ & & & \\
\hline & & & TNF-a & $569 \pm 45$ & 0 & & & \\
\hline
\end{tabular}

will contain or clear infections. However, if this response is continually activated, it may lead to lesion development and disease. It is still a matter of debate how spirochetes pass the blood-brain barrier, but hematogenous dissemination appears to be a suitable way [2]. Once they enter the $\mathrm{CNS}, \mathrm{Bb}$ encounter immune cells such as monocytes, macrophages, or dendritic cells, as well as glial cells such as microglia and astrocytes, all of which produce pro-inflammatory cytokines, e.g., IL-6, IL-8, IL-12, IL-18, and IFN $\gamma$, and
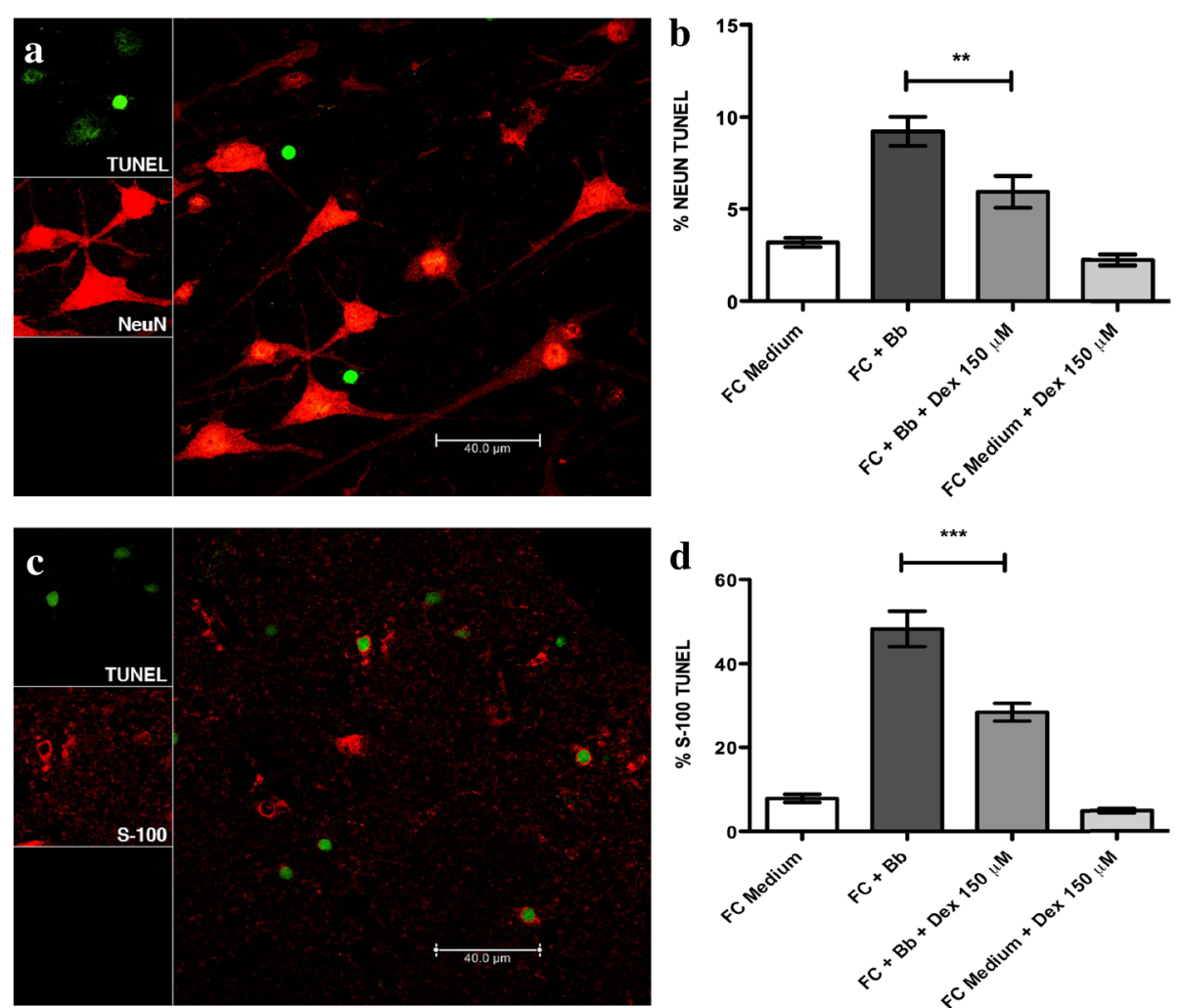

Fig. 3 Dexamethasone protects FC neurons $(\mathbf{a}, \mathbf{b})$ and oligodendrocytes (c, $\mathbf{d})$ from Bb-induced apoptosis. Representative images of apoptosis after immunofluorescence staining and visualized by confocal microscopy by the in situ TUNEL assay (green) in a neurons stained with NeuN $($ red), and c oligodendrocytes stained with S-100 (red). b, d Graphical representations of the effect of dexamethasone on the levels of apoptosis in neurons and oligodendrocytes, respectively, as induced by live Bb. The two-way ANOVA and Tukey's multiple comparison test were used to evaluate the statistical significance between means and SD of ten data sets (approximately 500 cells) for each condition, ${ }^{* *} p<0.01,{ }^{* * *} p<0.001$ 

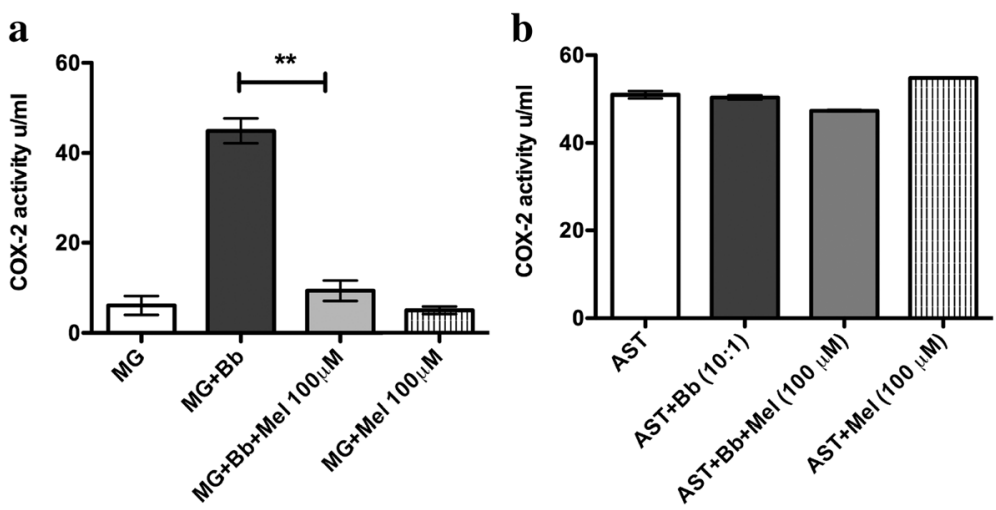

Fig. 4 Meloxicam's effect on COX-2 levels induced by live Bb in rhesus: a microglia and $\mathbf{b}$ astrocytes after 24 h of incubation. The two-way ANOVA and Tukey's multiple comparison test were used to evaluate the statistical significance between means and SEM of triplicate data sets for microglia and astrocytes, ${ }^{* *} p<0.01$

chemokines such as I-TAC, CCL2, CXCL-11, and CXCL13, as found in the CSF of patients [26-30] and rhesus macaques with $\operatorname{LNB}[11,14]$. In this study, we have demonstrated, with CNS cells and tissues, that a continuous inflammatory response is detrimental and leads to neural cell death.

Previously, we have shown that meloxicam was not a viable adjunctive therapeutic agent for the treatment of Bb-induced inflammation [11, 13]. Our present ex vivo and in vitro experiments (Fig. 1 and Table 1) confirm this finding. Meloxicam is analgesic and antipyretic; has a high gastric and renal tolerance; a high therapeutic index; and preferentially inhibits COX-2 [31]. To confirm that meloxicam effectively acted as a COX-2 inhibitor at the concentrations used in our study, we evaluated if this drug could dampen the level of this enzyme, as induced by live $\mathrm{Bb}$ in cell lysates of primary rhesus astrocytes and microglia. As shown in Fig. 4, meloxicam significantly reduced COX-2 levels from Bbinfected microglia (Fig. 4a), while it was unable to alter the constitutive levels of COX-2 in astrocytes (Fig. 4b). This verifies, on the one hand, that COX-2 is not causally involved in the inflammation by microglia in response to $\mathrm{Bb}$, as indicated by the lack of microglial antiinflammatory response to meloxicam, and on the other, that astrocytes do not respond to $\mathrm{Bb}$ by elevating the expression of COX-2.

Antibiotics will continue to be the first-line therapy for Lyme borreliosis. Steroids have been administered alongside antibiotics, with the literature reporting some beneficial effects [32, 33]. However, opposite results have been obtained as well in cases of facial palsy associated with Lyme neuroborreliosis [34]. Thus far, the findings from our group included in vivo, ex vivo, and in vitro works that used different neuronal and glial cells and suggested a protective role of dexamethasone in LNB. However, the implications of dexamethasone with regard to the treatment of human disease are not clear. What is known is that dexamethasone interferes with proinflammatory signal transduction, gene expression, and protein synthesis at various levels $[35,36]$. The actions of dexamethasone in bacterially induced TLR-mediated pro-inflammatory signaling involve inhibition of $\mathrm{I} \kappa \mathrm{B} \alpha$ phosphorylation and degradation as well as $N F-\kappa B$ DNA-binding activity [35]. Moreover, MEK, JNK, and p38, all belonging to the family of MAPKs, are prominent targets of dexamethasone [37-40]. A recent study from our group showed that the TLR2 pathway plays a predominant role in inducing CNS cell inflammation in response to $\mathrm{Bb}$ and that the downstream signaling involves the MyD88 and MAPK pathways [41]. Accordingly, it is possible that dexamethasone inhibits Bbinduced TLR2 signaling and the MEK/ERK pathway, reducing inflammation and subsequent apoptosis. Overall, our data suggest that inflammation has a causal role in the pathogenesis of CNS LNB. Further evaluation of its signaling and immunomodulatory mechanisms is still required to ascertain which inhibitors of inflammation may be safely used to mitigate the signs and symptoms of LNB.

\section{Conclusions}

These results indicate that dexamethasone and meloxicam have differential anti-inflammatory effects on Bbinduced inflammation in glial and neuronal cells of the CNS and help explain the in vivo findings of significantly reduced inflammatory mediators in the CSF and lack of inflammatory neurodegenerative lesions in the brain and spinal cord of Bb-infected animals that were treated with dexamethasone but not meloxicam. Signaling cascades altered by dexamethasone could serve as possible therapeutic targets for limiting CNS inflammation and tissue damage in LNB. 


\section{Abbreviations}

Bb: Borrelia burgdorferi; BSK: Barbour-Stoenner-Kelly; COX: Cyclooxygenase; CSF: Cerebrospinal fluid; ELISA: Enzyme-linked immunosorbent assay; LNB: Lyme neuroborreliosis; MBP: Myelin basic protein; MOI: Multiplicity of infection; MTT: 3-(4,5-dimethylthiazol-2-yl)-2,5-diphenyltetrazolium bromide; PBS: Phosphate-buffered saline; PNS: Peripheral nervous system; TUNEL: Terminal deoxynucleotidyl transferase dUTP nick end labeling

\section{Acknowledgements}

We thank the TNPRC Pathogen Detection and Quantification Core Laboratory for the help with the multiplex ELISA assays. Robin Rodriguez from the TNPRC Media Laboratory is gratefully acknowledged for assisting with the formatting of figures.

\section{Funding}

This project was supported by the National Institute of Neurologic Disorders and Stroke, through grant NS048952, and by the National Center for Research Resources and the Office of Research Infrastructure Programs (ORIP) of the National Institutes of Health through grant P51OD011104/P51RR000164.

\section{Availability of data and materials}

Please contact author for data requests.

\section{Authors' contributions}

GR participated in the design of the experiments, conducted the experiments, and wrote part of the first draft of the manuscript. ANM contributed to the rhesus FC explant tissue processing and ELISA experiments and completed the writing of the manuscript. DSM contributed to the immunofluorescence staining for confocal microscopy. MTP conceived the study, contributed to the design of the experiments and to the drafting and editing of the manuscript. All authors have read and approved the final version of the manuscript.

\section{Competing interests}

The authors declare that they have no competing interests.

\section{Consent for publication}

Not applicable.

\section{Ethics approval and consent to participate}

Not applicable.

Received: 3 November 2016 Accepted: 26 January 2017

Published online: 02 February 2017

\section{References}

1. Steere AC. Lyme disease. N Engl J Med. 2001;345:115-25.

2. Rupprecht TA, Koedel U, Fingerle V, Pfister H-W. The pathogenesis of lyme neuroborreliosis: from infection to inflammation. Mol Med. 2008:14:205-12.

3. Philipp MT, Aydintug MK, Bohm RP, Cogswell FB, Dennis VA, Lanners HN, Lowrie RC, Roberts ED, Conway MD, Karaçorlu M, Peyman GA, Gubler DJ, Johnson BJ, Piesman J, Gu Y. Early and early disseminated phases of Lyme disease in the rhesus monkey: a model for infection in humans. Infect Immun. 1993;61:3047-59.

4. Roberts ED, Bohm RP, Cogswell FB, Lanners HN, Lowrie RC, Povinelli L, Piesman J, Philipp MT. Chronic lyme disease in the rhesus monkey. Lab Invest. 1995;72:146-60

5. Cadavid D, O'Neill T, Schaefer H, Pachner AR. Localization of Borrelia burgdorferi in the nervous system and other organs in a nonhuman primate model of lyme disease. Lab Invest. 2000;80:1043-54.

6. Ramesh G, Borda JT, Gill A, Ribka EP, Morici LA, Mottram P, Martin DS, Jacobs MB, Didier PJ, Philipp MT. Possible role of glial cells in the onset and progression of Lyme neuroborreliosis. J Neuroinflammation. 2009;6:23.

7. Fallon BA, Levin ES, Schweitzer PJ, Hardesty D. Inflammation and central nervous system Lyme disease. Neurobiol Dis. 2010;37:534-41.

8. Vallat JM, Hugon J, Lubeau M, Leboutet MJ, Dumas M, Desproges-Gotteron R. Tick-bite meningoradiculoneuritis: clinical, electrophysiologic, and histologic findings in 10 cases. Neurology. 1987;37:749-53.

9. Payne DN, Adcock IM. Molecular mechanisms of corticosteroid actions. Paediatr Respir Rev. 2001;2:145-50.
10. Furst DE. Meloxicam: selective COX-2 inhibition in clinical practice. Semin Arthritis Rheum. 1997;26(6 Suppl 1):21-7.

11. Ramesh G, Didier PJ, England JD, Santana-Gould L, Doyle-Meyers LA, Martin DS, Jacobs MB, Philipp MT. Inflammation in the pathogenesis of Lyme neuroborreliosis. Am J Pathol. 2015;185:1344-60.

12. Ramesh G, Santana-Gould L, Inglis FM, England JD, Philipp MT. The Lyme disease spirochete Borrelia burgdorferi induces inflammation and apoptosis in cells from dorsal root ganglia. J Neuroinflammation. 2013;10:88.

13. Ramesh G, Meisner OC, Philipp MT. Anti-inflammatory effects of dexamethasone and meloxicam on Borrelia burgdorferi-induced inflammation in neuronal cultures of dorsal root ganglia and myelinating cells of the peripheral nervous system. J Neuroinflammation. 2015;12:240.

14. Ramesh G, Borda JT, Dufour J, Kaushal D, Ramamoorthy R, Lackner AA, Philipp MT. Interaction of the Lyme disease spirochete Borrelia burgdorferi with brain parenchyma elicits inflammatory mediators from glial cells as well as glial and neuronal apoptosis. Am J Pathol. 2008;173:1415-27.

15. Bernardino ALF, Myers TA, Alvarez X, Hasegawa A, Philipp MT. Toll-like receptors: insights into their possible role in the pathogenesis of lyme neuroborreliosis. Infect Immun. 2008;76:4385-95.

16. Ramesh G, Benge S, Pahar B, Philipp MT. A possible role for inflammation in mediating apoptosis of oligodendrocytes as induced by the Lyme disease spirochete Borrelia burgdorferi. J Neuroinflammation. 2012;9:72.

17. Porcella SF, Schwan TG. Borrelia burgdorferi and Treponema pallidum: a comparison of functional genomics, environmental adaptations, and pathogenic mechanisms. J Clin Invest. 2001;107:651-6.

18. Zhou Y, Ling E-A, Dheen ST. Dexamethasone suppresses monocyte chemoattractant protein-1 production via mitogen activated protein kinase phosphatase-1 dependent inhibition of Jun N-terminal kinase and p38 mitogen-activated protein kinase in activated rat microglia. J Neurochem. 2007;102:667-78.

19. Engelhardt G, Bögel R, Schnitzer C, Utzmann R. Meloxicam: influence on arachidonic acid metabolism. Part 1. In vitro findings. Biochem Pharmacol. 1996;51:21-8

20. Myers TA, Kaushal D, Philipp MT. Microglia are mediators of Borrelia burgdorferi-induced apoptosis in SH-SY5Y neuronal cells. PLOS Pathog. 2009:5:e1000659.

21. Minghetti L. Cyclooxygenase-2 (COX-2) in inflammatory and degenerative brain diseases. J Neuropathol Exp Neurol. 2004;63:901-10.

22. Djukic M, Schmidt-Samoa C, Lange P, Spreer A, Neubieser K, Eiffert H, Nau R, Schmidt H. Cerebrospinal fluid findings in adults with acute Lyme neuroborreliosis. J Neurol. 2012;259:630-6.

23. Bremell D, Hagberg L. Clinical characteristics and cerebrospinal fluid parameters in patients with peripheral facial palsy caused by Lyme neuroborreliosis compared with facial palsy of unknown origin (Bell's palsy). BMC Infect Dis. 2011:11:215.

24. Hodzic E. Lyme Borreliosis: is there a preexisting (natural) variation in antimicrobial susceptibility among Borrelia burgdorferi strains? Bosn J Basic Med Sci. 2015;15:1-13

25. Liang FT, Nelson FK, Fikrig E. DNA microarray assessment of putative Borrelia burgdorferi lipoprotein genes. Infect Immun. 2002;70:3300-3.

26. Cepok S, Zhou D, Vogel F, Rosche B, Grummel V, Sommer N, Hemmer B. The immune response at onset and during recovery from Borrelia burgdorferi meningoradiculitis. Arch Neurol. 2003:60:849-55.

27. Grusell M, Widhe M, Ekerfelt C. Increased expression of the Th1inducing cytokines interleukin-12 and interleukin-18 in cerebrospinal fluid but not in sera from patients with Lyme neuroborreliosis. J Neuroimmunol. 2002;131:173-8.

28. Widhe M, Grusell M, Ekerfelt C, Vrethem M, Forsberg P, Ernerudh J. Cytokines in Lyme borreliosis: lack of early tumour necrosis factor-alpha and transforming growth factor-beta1 responses are associated with chronic neuroborreliosis. Immunology. 2002;107:46-55.

29. Cerar T, Ogrinc K, Lotric-Furlan S, Kobal J, Levicnik-Stezinar S, Strle F, RuzićSabljic E. Diagnostic value of cytokines and chemokines in lyme neuroborreliosis. Clin Vaccine Immunol. 2013;20:1578-84.

30. Ramesh G, Maclean AG, Philipp MT. Cytokines and chemokines at the crossroads of neuroinflammation, neurodegeneration, and neuropathic pain. Mediators Inflamm. 2013;2013:480739.

31. laniski FR, Alves CB, Ferreira CF, Rech VC, Savegnago L, Wilhelm EA, Luchese C. Meloxicam-loaded nanocapsules as an alternative to improve memory decline in an Alzheimer's disease model in mice: involvement of $\mathrm{Na}$ K+ -ATPase. Metab Brain Dis. 2016:31:793-802. 
32. Nimmrich S, Becker I, Horneff G. Intraarticular corticosteroids in refractory childhood Lyme arthritis. Rheumatol Int. 2014;34:987-94.

33. Massengo SA, Bonnet F, Braun C, Vital A, Beylot J, Bastard J. Severe neuroborreliosis: the benefit of prolonged high-dose combination of antimicrobial agents with steroids - an illustrative case. Diagn Microbiol Infect Dis. 2005;51:127-30.

34. Jowett N, Gaudin RA, Banks CA, Hadlock TA: Steroid use in Lyme diseaseassociated facial palsy is associated with worse long-term outcomes. Laryngoscope. 2016. doi:10.1002/lary.26273.

35. Mogensen TH, Berg RS, Paludan SR, Østergaard L. Mechanisms of dexamethasone-mediated inhibition of Toll-like receptor signaling induced by Neisseria meningitidis and Streptococcus pneumoniae. Infect Immun. 2008;76:189-97.

36. Jang B-C, Lim K-J, Suh M-H, Park J-G, Suh S-I. Dexamethasone suppresses interleukin-1 beta-induced human beta-defensin 2 mRNA expression: involvement of p38 MAPK, JNK, MKP-1, and NF-kappaB transcriptional factor in A549 cells. FEMS Immunol Med Microbiol. 2007;51:171-84.

37. Sakai A, Han J, Cato ACB, Akira S, Li J-D. Glucocorticoids synergize with IL-1 beta to induce TLR2 expression via MAP Kinase Phosphatase-1dependent dual Inhibition of MAPK JNK and p38 in epithelial cells. BMC Mol Biol. 2004;5:2.

38. Bhattacharyya S, Brown DE, Brewer JA, Vogt SK, Muglia LJ. Macrophage glucocorticoid receptors regulate Toll-like receptor 4-mediated inflammatory responses by selective inhibition of p38 MAP kinase. Blood. 2007;109:4313-9.

39. Morita M, Suyama H, Igishi T, Shigeoka Y, Kodani M, Hashimoto K, Takeda K, Sumikawa T, Shimizu E. Dexamethasone inhibits paclitaxel-induced cytotoxic activity through retinoblastoma protein dephosphorylation in non-small cell lung cancer cells. Int J Oncol. 2007;30:187-92.

40. Pelaia G, Vatrella A, Cuda G, Maselli R, Marsico SA. Molecular mechanisms of corticosteroid actions in chronic inflammatory airway diseases. Life Sci. 2003; 72:1549-61.

41. Parthasarathy G, Philipp MT. Inflammatory mediator release from primary rhesus microglia in response to Borrelia burgdorferi results from the activation of several receptors and pathways. J Neuroinflammation. 2015;12:60.

\section{Submit your next manuscript to BioMed Central and we will help you at every step:}

- We accept pre-submission inquiries

- Our selector tool helps you to find the most relevant journal

- We provide round the clock customer support

- Convenient online submission

- Thorough peer review

- Inclusion in PubMed and all major indexing services

- Maximum visibility for your research

Submit your manuscript at www.biomedcentral.com/submit

) Biomed Central 